\title{
Il ruolo degli alleli del recettore della vitamina D nell'iperparatiroidismo secondario dei pazienti in emodialisi
}

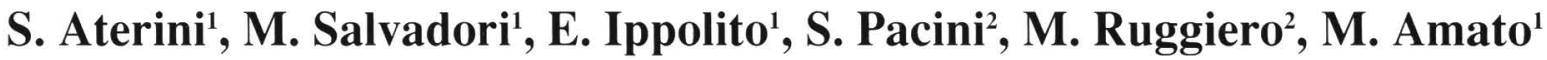 \\ ${ }^{1}$ U.O. Nefrologia e Dialisi, Ospedale di Prato \\ ${ }^{2}$ Istituto di Patologia Generale, Università di Firenze
}

insufficiente escrezione dei fosfati è stata indicata come la causa principale della ipersecrezione di ormone paratiroideo (PTH) osservata in corso di uremia. Sebbene 1'accumulo di fosfati svolga indubbiamente un ruolo importante nella patogenesi della patologia ossea in corso di malattie renali, recentemente è stato sottolineato come la ridotta capacità da parte del rene di sintetizzare il calcitriolo possa rappresentare un fattore importante nella patogenesi della osteodistrofia renale (1-3). L'influenza dei fattori genetici sulla massa ossea è nota da tempo, ma solo recentemente è stato sottolineato il ruolo svolto dal polimorfismo del gene per il recettore della vitamina $\mathrm{D}$ (VDR) nel determinare la massa ossea (4): l'allele "b" del gene per il VDR è associato con una densità ossea (BMD) superiore rispetto al genotipo BB. Il gene per il recettore della vitamina $\mathrm{D}$ è il principale regolatore della massa ossea, agendo attraverso fattori genetici e non (assorbimento del calcio alimentare responsabile del picco di massa ossea). Tuttavia i risultati concernenti l'impiego della vitamina D nel trattamento dell'osteoporosi sono controversi (5-7). Abbiamo quindi cercato di valutare se fra i pazienti emodializza- ti, in trattamento o meno con calcitriolo, esistessero differenze collegate agli alleli per il VDR. La valutazione del gene del VDR può essere utile per una migliore comprensione della sensibilità alla terapia di ogni paziente, poiché una predisposizione genetica alla bassa densità ossea potrebbe essere mitigata da un trattamento precoce ed efficace. Abbiamo così effettuato una serie di misurazioni di densità ossea, ad intervalli di tempo, per valutare il miglioramento o peggioramento della BMD. I nostri risultati suggeriscono che la terapia con vitamina D può prevenire, o almeno rallentare, la perdita di massa ossea nei pazienti in dialisi che condividono lo "stress" della uremia e una predisposizione genetica. Le variazioni funzionali del VDR possono inoltre essere utili nella interpretazione della variabilità dei livelli circolanti di paratormone osservata nei pazienti in trattamento emodialitico.

\section{Metodi}

Sono stati studiati 30 pazienti in trattamento con bicarbonato dialisi standard (sei donne premenopausali e 24 maschi). Nessuno era affetto da diabete mellito, né aveva ricevuto terapia steroidea, né era stato paratiroidectomizzato. Ognuno riceveva calcitriolo endovena alla fine della seduta dialitica (2-3 $\mu$ g alla settimana). Come chelante del fosforo era impiegato il calcio carbonato (4-8 $\mathrm{g}$ al giorno). L'acqua era trattata con osmosi inversa; il bagno dialisi conteneva calcio $3.0 \mathrm{mEq} / \mathrm{L}$ e magnesio $0.5 \mathrm{mEq} / \mathrm{L}$. La concentrazione dell'alluminio nel bagno dialisi era inferiore ai $10 \mu \mathrm{g} / \mathrm{L}$. L'adeguatezza del trattamento dialitico era valutata mediante determinazione della TAC urea.

Campioni di sangue erano prelevati all'inizio della dialisi per la misurazione di calcio, fosforo, fosfatasi alcalina (AP), alluminio, PTH e per l'estrazione del DNA. L'analisi genetica è stata condotta con metodica PCR della regione di DNA a fianco del sito di restrizione BsmI. La densiotometria ossea è stata misurata mediante absorbimetria a raggi $\mathrm{X}$ a doppia energia (DEXA), a livello della spina lombare (L1-L5), poiché in questa sede, in accordo ai più recenti studi, è più evidente l'influenza del fattore genetico.

Le variabili nei differenti genotipi sono state paragonate impiegando i test statistici non-parametrici di Mann-Whitney e di Kruskal-Wallis. Erano considerati si- 


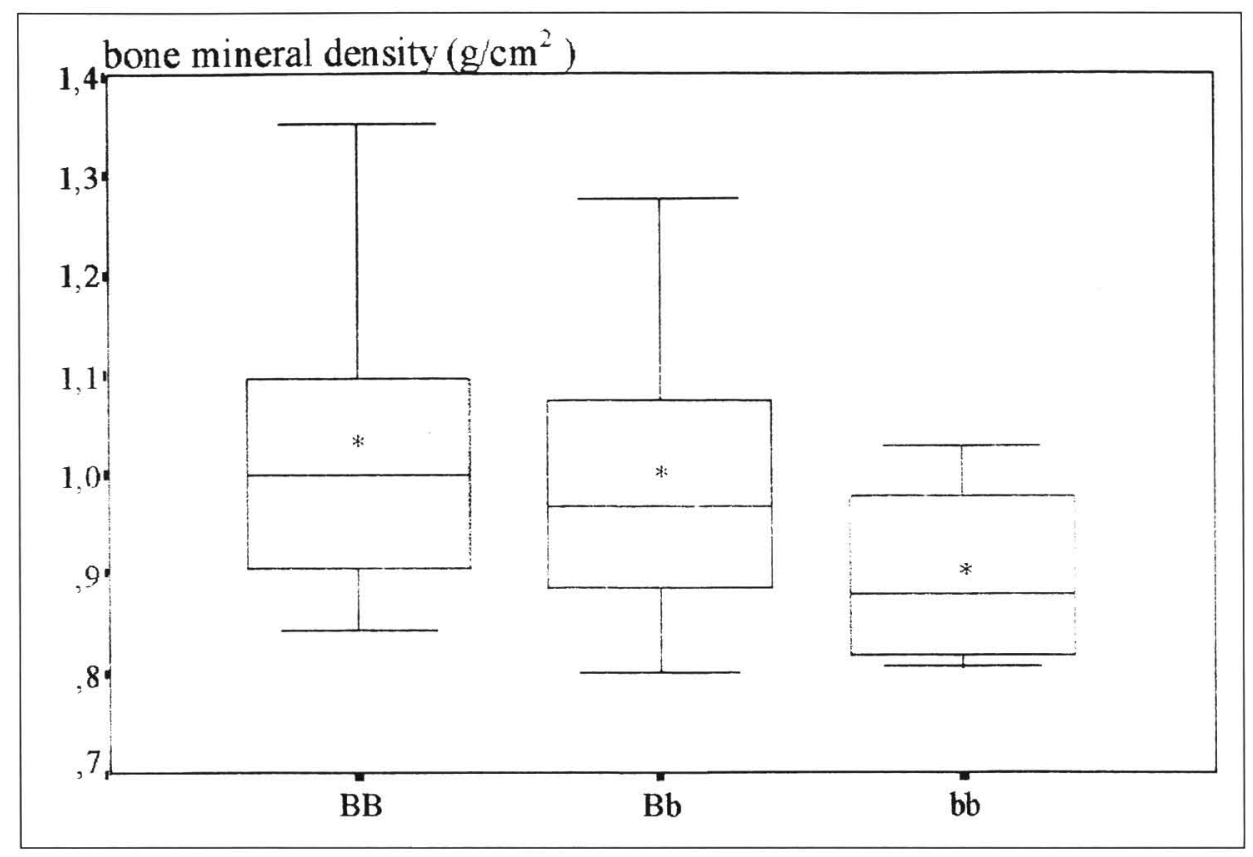

Fig. 1 - Densità minerale ossea a livello del rachide lombare nei pazienti dializzati, secondo il genotipo VDR. I rettangoli rappresentano il 50\% dei casi; la mediana è indicata dalla barra trasversale; la media è rappresentata dall' asterisco.

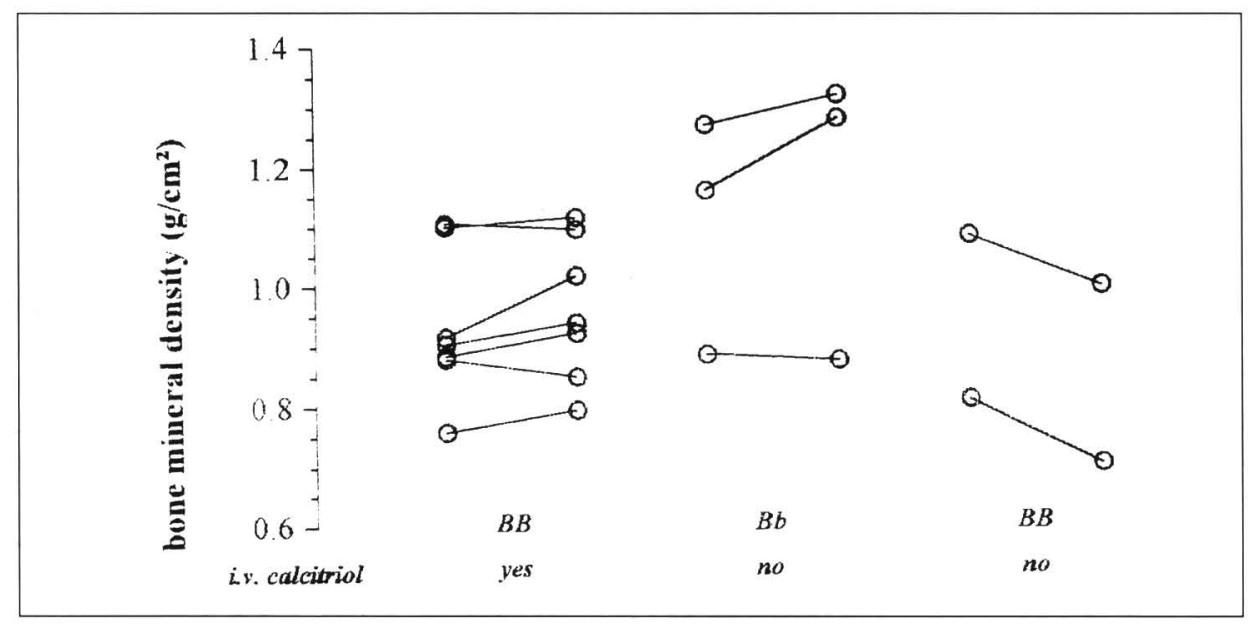

Fig. 2 - Genotipo del recettore per la vitamina $D$ e densità ossea a livello del rachide lombare, secondo il trattamento con calcitriolo. Le linee collegano $i$ valori di BMD basali e quelli ottenuti a distanza di un anno.

gnificativi i valori di p inferiori allo 0.05 .

\section{Risultati}

Le caratteristiche dei pazienti, suddivisi sulla base dei diversi genotipi per il VDR, al momento della prima misurazione di BMD sono illustrate nella Tabella I. Non c'era nessuna differenza fra i tre gruppi per quanto riguardava l'età, il periodo trascorso in dialisi, le concentrazioni sieriche di calcio, fosforo, fosfatasi alcalina e PTH. I livelli plasmatici di alluminio erano leggermente al di sopra dei valori di riferimento. Il trattamento dialitico era adeguato in tutti i pazienti. Nella Figura 1 sono riportati i valori di BMD misurati nei tre genotipi. La densità ossea era inferiore rispetto a quella dei controlli di pari età e sesso: bb -24 \pm $5 \%$; Bb $-17 \pm 3 \%$; BB $-15 \pm 5 \%$ (media \pm ES). La densità ossea era comunque entro l'intervallo normale (range: donne 0.780 - $1.375 \mathrm{~g} / \mathrm{cm}^{2}$; uomini 0.946 $1.508 \mathrm{~g} / \mathrm{cm}^{2}$ ). Sebbene i pazienti con genotipo bb avessero una BMD mediamente inferiore, le differenze fra i tre gruppi non erano statisticamente significative. La densità ossea non era correlata con il periodo di dialisi né con i valori di PTH circolante. Al fine di valutare l'influenza della componente genetica e della somministrazione di calcitriolo sulla massa ossea, la BMD è stata controllata dodici mesi dopo la prima valutazione in sette pazienti con genotipo BB trattati con calcitriolo e.v. e nessuna significativa variazione era presente (Fig. 2). Al contrario, due pazienti con il medesimo genotipo che non assumevano calcitriolo mostravano una netta riduzione della densità ossea a distanza di un anno (Fig. 2). I pazienti con genotipo eterozigote $\mathrm{Bb}$ non mostravano significative variazioni nel tempo della BMD, indipendentemente dal trattamento con calcitriolo (Fig. 2). Le concentrazioni sieriche del paratormone erano positivamente correlate con $\mathrm{i}$ valori di fosfatasi alcalina $(r=0.52$; $\mathrm{p}<$ 0.001 ), ma non con l'età dei pazienti né con il tempo trascorso in dialisi. Prima dell'inizio della terapia con calcitriolo; i pazienti con genotipo bb presentavano le più alte concentrazioni di PTH (465 \pm $112 \mathrm{pg} / \mathrm{ml}$; media $\pm \mathrm{DS}$ ), mentre quelli che condividevano gli alleli $\mathrm{BB}$ mostravano valori inferiori di PTH $(252 \pm 49$ $\mathrm{pg} / \mathrm{ml}$ ); livelli intermedi erano presenti nei soggetti con genotipo eterozigote $(\mathrm{Bb}$ $(408 \pm 48 \mathrm{pg} / \mathrm{ml})$. Al momento della misurazione della BMD i livelli del PTH nei tre gruppi di pazienti non presentavano significative differenze (Tab. I). Tuttavia, in seguito al trattamento con calcitriolo le concentrazioni sieriche di PTH presentavano una progressiva riduzione con un andamento diverso nei tre genotipi, con una riduzione, dopo 48 mesi di terapia, prossima al $60 \%$ nei pazienti portatori dell'allele "b", mentre i soggetti omozigoti BB mostravano una risposta al calcitriolo più lenta (Fig. 4).

\section{Discussione}

In questo studio abbiamo indagato la relazione fra il genotipo per il VDR, la densità minerale ossea e il paratormone. Studi recenti suggeriscono che le tossine uremiche riducono la interazione del complesso calcitriolo-VDR con il DNA 

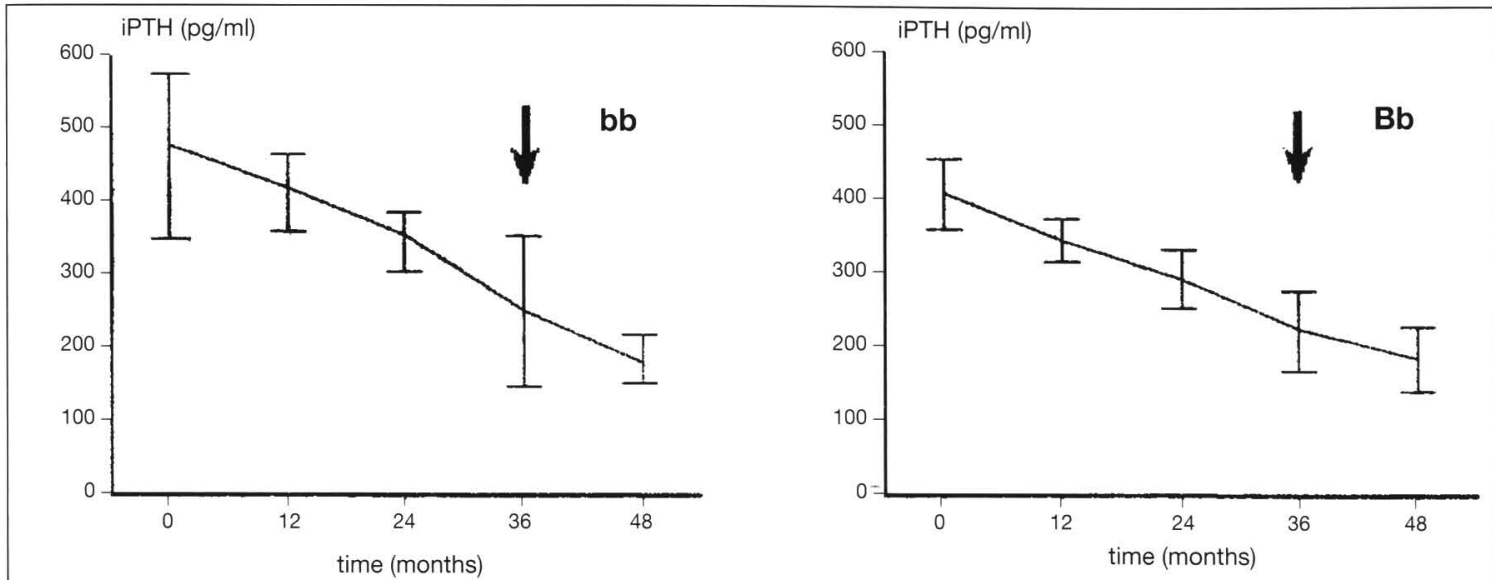

Fig. 3 - Concentrazioni plasmatiche di PTH nei tre genotipi del VDR nei pazienti emodializzati. Le frecce indicano il momento della prima misurazione di BMD.

Fig. 4 - Risposta del PTH alla terapia con calcitriolo e.v., espressa come variazione percentuale al valore basale, nei diversi genotipi del $V D R(* P<0.01$ vs bb e Bb).

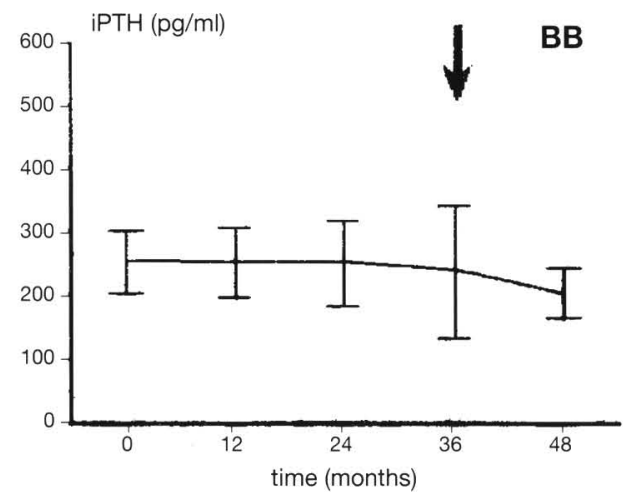

$(3,8-10)$ e che la concentrazione intracellulare del VDR è ridotta nell'insufficienza renale cronica $(11,12)$. Alcune recenti osservazioni suggeriscono che il genotipo bb sia più sensibile alla azione regolatrice da parte della vitamina $\mathrm{D}$ circolante $(13,14)$. Questa ipotesi è anche suffragata dalla nostra osservazione che i pazienti con questo genotipo mostravano

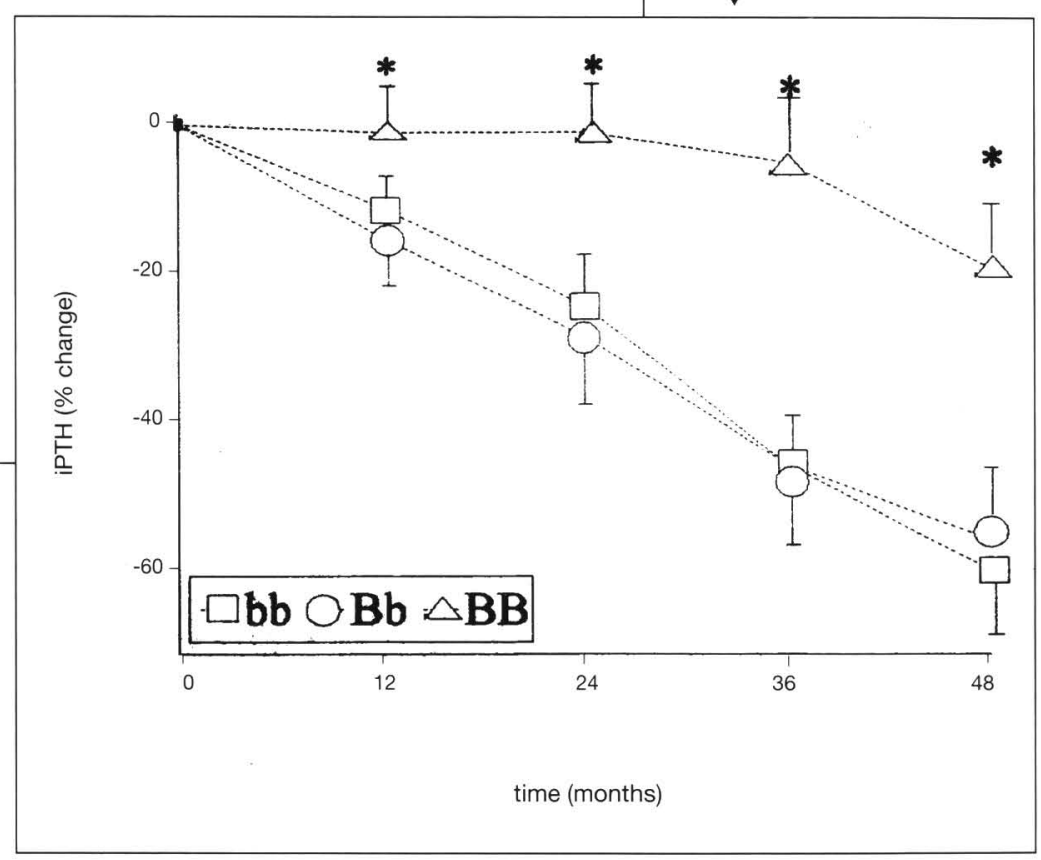

TABELLA I - CARATTERISTICHE CLINICHE DEI PAZIENTI AL MOMENTO DELLA PRIMA MISURAZIONE DELLA BMD

\begin{tabular}{ccccc}
\hline & $b b$ & $B b$ & BB & \\
\cline { 2 - 4 } M/F & $3 / 1$ & $15 / 4$ & $6 / 1$ \\
\hline
\end{tabular}

Mean (95\% Confidence intervals)

$\mathrm{p}$

Age $(\mathrm{yr})$

$\begin{array}{cl}44 & (21-64) \\ 52 & (14-108) \\ 9.3 & (8.1-10.4) \\ 4.8 & (3.3-6.3) \\ 253 & (140-379) \\ 14 & (6-26) \\ 253 & (179-441) \\ 42 & (34-49)\end{array}$

$\begin{array}{cl}52 & (43-61) \\ 56 & (28-113) \\ 10.0 & (9.6-10.4) \\ 4.7 & (4.3-5.1) \\ 254 & (190-318) \\ 18 & (11-24) \\ 223 & (100-346) \\ 42 & (35-50)\end{array}$

$57(47-67)$

n.s.

$50 \quad(35-64)$

$9.4 \quad(8.5-10.2)$

n.s.

$\mathrm{Ca}(8.5-10.5 \mathrm{mg} / \mathrm{dl})$

$\mathrm{P}(2.4-4.8 \mathrm{mg} / \mathrm{dl})$

AP (70 - $280 \mathrm{U} / \mathrm{L})$

$\mathrm{Al}(<10 \mu \mathrm{g} / \mathrm{L})$

iPTH $(0-55$ pg/ml)

TAC urea (40 - 60)
42 (34-49)
$5.4(4.9-5.5)$

$223(164-282)$

14 (8 - 18)

$238 \quad(182-359)$

$39(32-46)$ n.s.

n.s.

n.s.

n.s.

n.s.

n.s. 
valori più elevati di PTH prima della terapia con vitamina $\mathrm{D}$. Inoltre nel presente studio sono state chiaramente identificate diversità di risposte alla terapia fra $\mathrm{i}$ vari genotipi che potrebbero fornire la interpretazione per i diversi gradi di iperparatiroidismo osservati in corso di insufficienza renale cronica (15-17), sebbene l'esatto meccanismo molecolare che ne è alla base resta da essere chiarito.

La densità ossea nei nostri pazienti era ridotta, ma sempre compresa nei limiti della norma. I ridotti valori di BMD nei pazienti con genotipo bb potrebbero essere in relazione al maggior grado di iperparatiroidismo presente in questo gruppo di soggetti. I pazienti con genotipo BB trattati regolarmente con calcitriolo non mostravano variazioni nel tempo della BMD, mentre quelli con il medesimo genotipo ma non trattati presentavano una rapida perdita ossea. I soggetti eterozigoti $\mathrm{Bb}$ erano invece protetti dalla presenza dell'allelle " $b$ " anche in assenza di terapia.

In conclusione, la relazione fra VDR e massa ossea nei pazienti dializzati resta da chiarire, anche se non si può escludere l'ipotesi che le anomalie nella sintesi del VDR e nella sua interazione con il DNA possano prevalere su di una potenziale protezione genetica. Infine, la valutazione genetica del VDR può aiutare nell'interpretazione della variabilità dei livelli circolanti di PTH e della differente sensibilità al trattamento.

\section{BIBLIOGRAFIA}

1. Llach F. Secondary hyperparathyroidism in renal failure. The trade-off hypothesis revisited. Am J Kidney Dis 1995; 25: 663-79.

2. Slatopolsky E, Berkoben M, Kelber J, Brown A, Delmez J. Effects of calcitriol and non-calcemic Vitamin D analogues on secondary hyperathyroidism. Kidney Int 1992; 42: (suppl 38): S43-9.

3. Hsu CH, Patel SR, Young EW, Vanholder R. The biological action of calcitriol in renal failure. Kidney Int 1994; 46: 605-12.

4. Morrison NA, Cheng QIJ. Prediction of bone density from calcitriol receptor alleles. Nature 1994; 367: 284-7.

5. Kanis JA. Osteoporosis. Oxford: Blackwell Science Ltd, 1994; 212.

6. Tilyard MW, Spears GFS, Thomson J, Dovey S. Treatment of postmenopausal osteoporosis with calcitriol or calcium. N Engl J Med 1992; 326: 357-62.

7. Chapuy MC, Arlot ME, Duboeuf F, et al. Vitamin D3 and calcium to prevent hip fractures in elderly women. N Engl J Med 1992; 327: $1637-42$.

8. Patel SR, Ke HQ, Hsu CH. Regulation of calcitriol receptor and its mRNA in normal and renal failure rats. Kidney Int 1994; 45: 1020-7.

9. Patel SR, Qiong $\mathrm{Ke} \mathrm{H}$, Vanholder R, Hsu CH. Inhibition of nuclear uptake of calcitriol receptor by uremic ultrafiltrate. Kidney Int 1994; 46: 129-33.

10. Hsu CH, Patel SR, Young EW. Mechanism of decresed calcitriol degradation in renal failure. Am J Physiol 1992; 262: F192-8.

11. Brown AJ, Dusso A, LopezHilker S, Lewis-Finch J, Grooms P,
Slatopolsky E. 1,25 $(\mathrm{OH})_{2} \mathrm{D} 3$ receptors are decreased in parathyroid glands from chronically uremic dogs. Kidney Int 1989; 35: 19-23.

12. Hsu CH, Patel RS, Vanholder R. Mechanism of decreased intestinal calcitriol receptor concentration in renal failure. Am J Physiol 1993; 264: F662-9.

13. Howard G, Nguyen T, Morrison $\mathrm{N}$, et al. Genetic influences on bone density: physiological correlates of vitamin D receptor gene alleles in premenopausal women. J Clin Endocrinol Metab 1995; 80: 2800-5.

14. Carling T, Kindmark A, Hellman $P$, et al. Vitamin D receptor genotypes in primary hyperparathyroidism. Nature Med 1995; 1: 1309-11.

15. Fukuda N, Tanaka H, Tominaga Y, Fukagawa M, Kurokawa K, Seino Y. Decreased 1,25-dihydroxycalcitriol 3 receptor density is associated with a more severe form of parathyroid hyperplasia in chronic uremic patients. J Clin Invest 1993; 92: 1436-43.

16. Arnold A, Brown MF, Urena P, Gaz RD, Sarfati E, Drueke TB. Monoclonality of parathyroid tumors in chronic renal failure and in primary parathyroid hyperplasia. J Clin Invest 1995; 95: 2047-53.

17. Falchetti A, Bale AF, Amorosi $A$, et al. Progression of uremic hyperparathyroidism involves allelic loss on chromosome 11. J Clin Endocrinol Metab 1993; 76: 139-44. 